\title{
Quantized orthonormal systems: A non-commutative Kwapień theorem
}

\author{
by \\ J. García-Cuerva and J. Parcet (Madrid)
}

\begin{abstract}
The concepts of Riesz type and cotype of a given Banach space are extended to a non-commutative setting. First, the Banach space is replaced by an operator space. The notion of quantized orthonormal system, which plays the role of an orthonormal system in the classical setting, is then defined. The Fourier type and cotype of an operator space with respect to a non-commutative compact group fit in this context. Also, the quantized analogs of Rademacher and Gaussian systems are treated. All this is used to obtain an operator space version of the classical theorem of Kwapien characterizing Hilbert spaces by means of vector-valued orthogonal series. Several approaches to this result with different consequences are given.
\end{abstract}

1. Introduction. The notion of type or cotype of a Banach space $B$ with respect to some classical system, such as the Rademacher or trigonometric system, is a common way to express the validity of certain inequalities for $B$-valued functions. The systematic investigation of these topics has given rise to a very well developed theory of the interaction between orthonormal systems and the geometry of Banach spaces. In this paper we look at this interaction from a non-commutative point of view. By that we mean to investigate what happens when we replace Banach spaces by operator spaces.

The first example in this setting was given in [5], where we define and study the Fourier type and cotype of an operator space with respect to a noncommutative compact group. Let $1 \leq p \leq 2$ and let $p^{\prime}$ denote its conjugate exponent. Let $G$ be a compact group with dual object $\Gamma$. An operator space $E$ has Fourier type $p$ with respect to $G$ if the $E$-valued Fourier transform on $G$ extends to a completely bounded operator from $L_{E}^{p}(G)$ into $\mathcal{L}_{E}^{p^{\prime}}(\Gamma)$. Similarly, by considering the inverse of the Fourier transform, the notion

2000 Mathematics Subject Classification: 46L07, 46C15, 42C15.

Key words and phrases: operator space, Kwapień theorem, quantized system, Riesz type, Rademacher system, Gauss system.

Research supported in part by the European Commission via the TMR Network 'Harmonic Analysis' and by Project BFM 2001/0189, Spain. 
of Fourier cotype comes out in this context. A relevant difference, between this notion of Fourier type and its classical counterpart for compact abelian groups, lies in the fact that the system of characters has to be replaced by the set of equivalence classes of unitary irreducible representations of $G$. That is, $\Gamma$ is now the system and $E$ is the space.

Going back to the general case, the question is to find which properties should be required of the system to get appropriate information about the operator space. As we shall see below, these systems will be collections of matrix-valued functions satisfying some extra conditions, which is perfectly natural in view of the basic example mentioned above. This is why we have called them "quantized systems", completing in such a way the scheme where Banach spaces become operator spaces and boundedness of operators is replaced by complete boundedness. Finally we point out that since vector-valued Schatten classes appear in vector-valued orthogonal series with respect to a quantized orthonormal system, our work does not make sense in Banach space theory. The reader is referred to [13] for more on this topic.

The definition of a quantized orthonormal system was motivated by the theory initiated in [5] and [4]. In fact, basic to its development is an operator space version of the isomorphic characterization of Hilbert spaces given by Kwapień in [6]. We provide three different approaches to this result. The first one is valid for any uniformly bounded quantized orthonormal system. The second one extends this result to non-uniformly bounded but complete quantized orthonormal systems. The third approach involves the quantization of the classical Gauss system, which fails to be complete or even uniformly bounded. This system also characterizes Pisier's OH Hilbertian operator spaces up to complete isomorphism and the proof of this fact follows the argument given in the first approach. However, we also show that Kwapień's original argument in [6] to link Rademacher and Gauss systems via the central limit theorem works in this context. Moreover, as we shall see, the use of this probabilistic approach has a remarkable advantage. Namely, it provides the result of Corollary 5.7. Roughly speaking, the latter says that when the quantized system we deal with takes values in arbitrarily large matrices, then the operator space version of Kwapień's theorem for the system also holds if we require only the boundedness of the operators involved, not the complete boundedness. Finally, an example is included and some open questions are posed.

Throughout this paper some basic notions of operator space theory and vector-valued Schatten classes will be assumed. The definitions and results about operator spaces that we will be using can be found in the book of Effros and Ruan [2], while for the Schatten classes the reader is referred to [13], where Pisier analyzes them in detail. 
Acknowledgments. We wish to thank Gilles Pisier for some valuable comments, specially for the proof of Theorem 5.9, which he communicated to us during a visit of the second author to Université Paris VI.

2. Uniformly bounded quantized orthonormal systems. The classical Hausdorff-Young inequality on the torus was generalized by F. Riesz in 1923 to any uniformly bounded orthonormal system. If one looks for extensions of this result to vector-valued functions, the notions of Riesz type, cotype and strong cotype of a Banach space come out naturally. These were defined in [3] with the aim to provide a general notion of type which would cover the classical (uniformly bounded) systems: Rademacher, Fourier, Walsh, etc. Here we introduce a "quantized version" of these notions. From now on, $M_{n}$ will stand for the vector space of $n \times n$ complex matrices and $S_{n}^{p}$ will denote the Schatten $p$-class over $M_{n}$.

Definition 2.1. Let $(\Omega, \mathcal{M}, \mu)$ be a probability measure space with no atoms and let $\mathbf{d}_{\Sigma}=\left\{d_{\sigma}: \sigma \in \Sigma\right\}$ be a family of positive integers, with $\Sigma$ an index set. A collection $\Phi=\left\{\varphi^{\sigma}: \Omega \rightarrow M_{d_{\sigma}}\right\}_{\sigma \in \Sigma}$ of matrix-valued functions with measurable entries is said to be a uniformly bounded quantized orthonormal system (u.b.q.o.s. for short) if the following conditions hold:

$$
\begin{gathered}
\int_{\Omega} \varphi_{i j}^{\sigma}(\omega) \overline{\varphi_{i^{\prime} j^{\prime}}^{\sigma^{\prime}}(\omega)} d \mu(\omega)=\frac{1}{d_{\sigma}} \delta_{\sigma \sigma^{\prime}} \delta_{i i^{\prime}} \delta_{j j^{\prime}}, \\
\sup _{\sigma \in \Sigma} \operatorname{ess} \sup _{\omega \in \Omega}\left\|\varphi^{\sigma}(\omega)\right\|_{S_{d_{\sigma}}^{\infty}}=\mathrm{M}_{\Phi}<\infty .
\end{gathered}
$$

The pair $\left(\Sigma, \mathbf{d}_{\Sigma}\right)$ will be called the set of parameters of $\Phi$. We say that $\Phi$ is complete when any function $f \in L^{2}(\Omega)$ can be written as

$$
f=\sum_{\sigma \in \Sigma} d_{\sigma} \operatorname{tr}\left(A^{\sigma} \varphi^{\sigma}\right) \quad \text { for some } A \in \prod_{\sigma \in \Sigma} M_{d_{\sigma}} .
$$

REMARK 2.2. Recall that if we take $\Sigma=\mathbb{N}$ and $d_{\sigma}=1$ for all $\sigma \in \Sigma$, we recover the classical definition of uniformly bounded orthonormal systems or complete orthonormal systems on $\Omega$. Also, if $\Omega$ is a compact topological group $G$ with normalized Haar measure $\mu$, then the dual object $\Gamma$ of $G$ is a u.b.q.o.s. The functions $\varphi^{\sigma}$ are irreducible unitary representations of $G, d_{\sigma}$ is the degree of $\varphi^{\sigma}$ and $\mathrm{M}_{\Gamma}=1$.

Let $1 \leq p<\infty$, let $E$ be an operator space and let $\Sigma$ be an index set as in Definition 2.1. Following the notation of [5] we define the spaces

$$
\begin{aligned}
\mathcal{L}_{E}^{p}(\Sigma) & =\left\{A \in \prod_{\sigma \in \Sigma} M_{d_{\sigma}} \otimes E:\|A\|_{\mathcal{L}_{E}^{p}(\Sigma)}=\left(\sum_{\sigma \in \Sigma} d_{\sigma}\left\|A^{\sigma}\right\|_{S_{d_{\sigma}}^{p}(E)}^{p}\right)^{1 / p}<\infty\right\}, \\
\mathcal{L}_{E}^{\infty}(\Sigma) & =\left\{A \in \prod_{\sigma \in \Sigma} M_{d_{\sigma}} \otimes E:\|A\|_{\mathcal{L}_{E}^{\infty}(\Sigma)}=\sup _{\sigma \in \Sigma}\left\|A^{\sigma}\right\|_{S_{d_{\sigma}}^{\infty}(E)}<\infty\right\},
\end{aligned}
$$


where we write $S_{n}^{p}(E)$ for the $E$-valued Schatten $p$-class over $M_{n}$. We use $\mathcal{L}^{p}(\Sigma)$ in the scalar-valued case. $\mathcal{L}_{E}^{p}(\Sigma)$ is endowed with its natural operator space structure (see [5] and Chapter 2 of [13] for the details). Now, if $\Phi$ is a u.b.q.o.s. and $\star$ stands for the adjoint operator, then the $\Phi$-transform and its inverse can be defined naturally as follows:

$$
\mathcal{F}_{\Phi}(f)^{\sigma}=\int_{\Omega} f(\omega) \varphi^{\sigma}(\omega)^{\star} d \mu(\omega), \quad \mathcal{F}_{\Phi}^{-1}(A)(\omega)=\sum_{\sigma \in \Sigma} d_{\sigma} \operatorname{tr}\left(A^{\sigma} \varphi^{\sigma}(\omega)\right)
$$

for $f: \Omega \rightarrow E$ and $A \in \prod_{\sigma \in \Sigma} M_{d_{\sigma}} \otimes E$. Note that when the system $\Phi$ is not complete, the inverse is not unique. Thus our notation is slightly abusive, but we shall keep it for concreteness.

We start with a version of the classical Riesz theorem for uniformly bounded quantized orthonormal systems.

Lemma 2.3. Let $1 \leq p \leq 2$ and let $p^{\prime}$ denote its conjugate exponent. Let $\Phi$ be a u.b.q.o.s. Then

$$
\left\|\mathcal{F}_{\Phi}\right\|_{\operatorname{cb}\left(L^{p}(\Omega), \mathcal{L}^{p^{\prime}}(\Sigma)\right)},\left\|\mathcal{F}_{\Phi}^{-1}\right\|_{\operatorname{cb}\left(\mathcal{L}^{p}(\Sigma), L^{p^{\prime}}(\Omega)\right)} \leq \mathrm{M}_{\Phi}^{2 / p-1}
$$

Proof. By the complex interpolation method for operator spaces, we just need to check the cases $p=1,2$. It follows from Lemma 1.7 of [13] that

$$
\left\|\mathcal{F}_{\Phi}\right\|_{\operatorname{cb}\left(L^{2}(\Omega), \mathcal{L}^{2}(\Sigma)\right)}=\sup _{n \geq 1}\left\|\mathcal{F}_{\Phi} \otimes I_{M_{n}}\right\|_{\mathcal{B}\left(L_{S_{n}^{2}}^{2}(\Omega), \mathcal{L}_{S_{n}^{2}}^{2}(\Sigma)\right)}
$$

with the obvious modifications for the inverse operator. Then the case $p=2$ is a consequence of the orthonormality of $\Phi$. That is, it follows from condition (a) in Definition 2.1. If $p=1$, then $\mathcal{F}_{\Phi}$ is defined on $L^{1}(\Omega)$, which is equipped with the max quantization. Moreover, $\mathcal{F}_{\Phi}^{-1}$ takes values in $L^{\infty}(\Omega)$, which is equipped with the min quantization. Therefore, by the quantizations we are working with, boundedness is equivalent to complete boundedness (see Section 3.3 of [2] for the details). But it is obvious that the stated inequalities hold for $p=1$ when the cb norm is replaced by the operator norm.

If $\Sigma_{0}$ is a finite subset of $\Sigma$, let $\Phi_{E}^{p}\left(\Sigma_{0}\right)=\operatorname{span}\left\{\varphi_{i j}^{\sigma}: \sigma \in \Sigma_{0}\right\} \otimes E$ regarded as a subspace of $L_{E}^{p}(\Omega)$ with its natural operator space structure. Also, let $\Phi_{0}=\left\{\varphi^{\sigma}: \Omega \rightarrow M_{d_{\sigma}}\right\}_{\sigma \in \Sigma_{0}}$ be the restriction of $\Phi$ to $\Sigma_{0}$. Then $\Phi_{0}$ is also a u.b.q.o.s. and Lemma 2.3 holds for $\Phi_{0}$.

Definition 2.4. Let $1 \leq p \leq 2$, let $p^{\prime}$ denote its conjugate exponent, and let $E$ be an operator space.

- $E$ is said to have Riesz type $p$ with respect to $\Phi$, or simply $\Phi$-type $p$, if

$$
\mathcal{K}_{1 p}(E, \Phi)=\sup \left\|\mathcal{F}_{\Phi_{0}}^{-1} \otimes I_{E}\right\|_{\operatorname{cb}\left(\mathcal{L}_{E}^{p}\left(\Sigma_{0}\right), \Phi_{E}^{p^{\prime}}\left(\Sigma_{0}\right)\right)}<\infty
$$

where the supremum is taken over the family of finite subsets $\Sigma_{0}$ of $\Sigma$. 
- $E$ is said to have Riesz cotype $p^{\prime}$ with respect to $\Phi$, or simply $\Phi$-cotype $p^{\prime}$, if

$$
\mathcal{K}_{2 p^{\prime}}(E, \Phi)=\sup \left\|\mathcal{F}_{\Phi_{0}} \otimes I_{E}\right\|_{\operatorname{cb}\left(\Phi_{E}^{p}\left(\Sigma_{0}\right), \mathcal{L}_{E}^{p^{\prime}}\left(\Sigma_{0}\right)\right)}<\infty .
$$

The supremum is taken again over the family of finite subsets $\Sigma_{0}$ of $\Sigma$.

- $E$ is said to have strong Riesz cotype $p^{\prime}$ with respect to $\Phi$, or simply strong $\Phi$-cotype $p^{\prime}$, if

$$
\mathcal{K}_{3 p^{\prime}}(E, \Phi)=\left\|\mathcal{F}_{\Phi, E}\right\|_{\operatorname{cb}\left(L_{E}^{p}(\Omega), \mathcal{L}_{E}^{p^{\prime}}(\Sigma)\right)}<\infty
$$

where $\mathcal{F}_{\Phi, E}$ denotes the extension of $\mathcal{F}_{\Phi} \otimes I_{E}$ to $L_{E}^{p}(\Omega)$.

REMARK 2.5. Note that if $E$ has $\Phi$-type $p$, then in particular there exists a positive constant $c$ such that

$$
\left\|\sum_{\sigma \in \Sigma_{0}} d_{\sigma} \operatorname{tr}\left(A^{\sigma} \varphi^{\sigma}\right)\right\|_{L_{E}^{p^{\prime}}(\Omega)} \leq c\left(\sum_{\sigma \in \Sigma_{0}} d_{\sigma}\left\|A^{\sigma}\right\|_{S_{d_{\sigma}}^{p}(E)}^{p}\right)^{1 / p}
$$

for any finite subset $\Sigma_{0}$ of $\Sigma$ and any $A \in \mathcal{L}_{E}^{p}\left(\Sigma_{0}\right)$. This condition is much closer to the classical notion of Riesz type. In fact, for $d_{\sigma}=1$ and $\Sigma_{0}=$ $\{1, \ldots, n\}$, we recover the classical definition. Analogous remarks hold for the Riesz cotype and strong Riesz cotype.

REMARK 2.6. We point out here that, as in the classical theory, a notion of strong Riesz type would be superfluous since it would coincide with that of Riesz type. The proof of this fact is an easy consequence of the density of the subspace of $\mathcal{L}_{E}^{p}(\Sigma)$ formed by the elements $A$ with finite support, that is, with $A^{\sigma} \neq 0$ only for finitely many $\sigma \in \Sigma$. In fact,

$$
\mathcal{K}_{1 p}(E, \Phi)=\left\|\mathcal{F}_{\Phi, E}^{-1}\right\|_{\operatorname{cb}\left(\mathcal{L}_{E}^{p}(\Sigma), L_{E}^{p^{\prime}}(\Omega)\right)} .
$$

Again as in the classical case, this equivalence is not necessarily valid for the cotype. Moreover, we have the obvious estimate $\mathcal{K}_{2 p^{\prime}}(E, \Phi) \leq \mathcal{K}_{3 p^{\prime}}(E, \Phi)$ for any u.b.q.o.s. $\Phi$, any operator space $E$ and any $1 \leq p \leq 2$. However, the $\Phi$-cotype is equivalent to the strong $\Phi$-cotype when $\Phi$ is complete. In this paper we shall be mainly concerned with the Riesz type and cotype. We have defined the strong Riesz cotype because, as we shall see below, it is the right notion for duality.

REMARK 2.7. Occasionally, we shall also use the notion of $\Psi$-type 2 and $\Psi$-cotype 2 for some quantized orthonormal systems $\Psi$ which fail to be uniformly bounded.

These definitions are illustrated in [5] and [4] where the Fourier type and cotype of an operator space with respect to a compact group are investigated. Namely, if $G$ is a compact group with dual object $\Gamma$, then Fourier type $p$ with respect to $G$ is nothing but $\Gamma$-cotype $p^{\prime}$ (or strong $\Gamma$-cotype $p^{\prime}$ since $\Gamma$ is complete in $L^{2}(G)$ by the Peter-Weyl theorem). Moreover, Fourier cotype 
$p^{\prime}$ with respect to $G$ coincides with $\Gamma$-type $p$. This conflict in terminology goes back to the commutative theory, where Fourier type $p$ with respect to the torus $\mathbb{T}$ means $\mathbb{Z}$-cotype $p^{\prime}$ (or equivalently strong $\mathbb{Z}$-cotype $p^{\prime}$ ); see [3].

In what follows we assume the reader is familiar with the properties of Fourier type and cotype stated in [5] and [4]. In fact, we omit the proof of the following results, since the arguments can be found there.

(a) Trivial exponents. Every operator space has Riesz type 1 and strong Riesz cotype $\infty$ with respect to any u.b.q.o.s. $\Phi$. Moreover, we have the estimates $\mathcal{K}_{11}(E, \Phi), \mathcal{K}_{2 \infty}(E, \Phi), \mathcal{K}_{3 \infty}(E, \Phi) \leq \mathrm{M}_{\Phi}$.

(b) Subspaces. The Riesz type is preserved when passing to subspaces. Moreover, $\mathcal{K}_{1 p}(F, \Phi) \leq \mathcal{K}_{1 p}(E, \Phi)$ for any closed subspace $F$ of $E$. The same holds for the Riesz cotype and strong Riesz cotype.

(c) Complex interpolation. Let $0<\theta<1$ and let $E_{0}$ and $E_{1}$ be operator spaces having $\Phi$-type $p_{0}$ and $p_{1}$ respectively. If $\left(E_{0}, E_{1}\right)$ is compatible for complex interpolation, then the interpolated operator space $\left(E_{0}, E_{1}\right)_{\theta}$ has $\Phi$-type $p_{\theta}=p_{0} p_{1}\left((1-\theta) p_{1}+\theta p_{0}\right)^{-1}$. In particular, the Riesz type $p$ becomes a stronger condition on a given operator space as $p$ approaches 2. Similar assertions hold for the Riesz cotype and strong Riesz cotype.

(d) Duality. $\mathcal{K}_{1 p}(E, \Phi)=\mathcal{K}_{3 p^{\prime}}\left(E^{\star}, \Phi\right)$ and $\mathcal{K}_{1 p}\left(E^{\star}, \Phi\right)=\mathcal{K}_{3 p^{\prime}}(E, \Phi)$. That is, Riesz type and strong Riesz cotype are dual notions.

(e) Local theory. If $d_{\mathrm{cb}}$ stands for the cb-distance between two operator spaces, then $\mathcal{K}_{1 p}\left(E_{2}, \Phi\right) \leq d_{\mathrm{cb}}\left(E_{1}, E_{2}\right) \mathcal{K}_{1 p}\left(E_{1}, \Phi\right)$. The same holds for the Riesz cotype and strong Riesz cotype.

(f) Degenerate case. Assume that the index set $\Sigma$ associated to the u.b.q.o.s. $\Phi$ is finite. Then

$$
\mathcal{K}_{1 p}(E, \Phi), \mathcal{K}_{2 p^{\prime}}(E, \Phi), \mathcal{K}_{3 p^{\prime}}(E, \Phi) \leq \mathrm{M}_{\Phi}\left(\sum_{\sigma \in \Sigma} d_{\sigma}^{2}\right)^{1 / p^{\prime}} .
$$

(g) Lebesgue spaces. Let $1 \leq q \leq \infty$. Let $(X, \mathcal{N}, \nu)$ be a $\sigma$-finite measure space. Then $L^{q}(X)$ has $\Phi$-type $\min \left(q, q^{\prime}\right)$ and strong $\Phi$-cotype $\max \left(q, q^{\prime}\right)$. Similar results hold for Schatten classes. Moreover, $L_{E}^{q}(X)$ and $S^{q}(E)$ have $\Phi$-type $\min \left(q, q^{\prime}\right)$ and strong $\Phi$-cotype $\max \left(q, q^{\prime}\right)$ whenever $E$ does.

REMARK 2.8. In what follows we shall assume that $\Sigma$ is not finite.

3. The Kwapień theorem for operator spaces. We begin by defining the quantized version of the classical Rademacher system. This notion is extracted from [10], where it is used to study random Fourier series on non-commutative compact groups. From now on we fix a probability measure space $(\Omega, \mathcal{M}, \mu)$ with no atoms, an index set $\Sigma$ and a family $\mathbf{d}_{\Sigma}$ of positive integers. 
DeFinition 3.1. The quantized Rademacher system associated to $\left(\Sigma, \mathbf{d}_{\Sigma}\right)$ is defined by a collection $\mathcal{R}=\left\{\varrho^{\sigma}: \Omega \rightarrow O\left(d_{\sigma}\right)\right\}_{\sigma \in \Sigma}$ of independent random orthogonal matrices, uniformly distributed on the orthogonal group $O\left(d_{\sigma}\right)$ equipped with its normalized Haar measure $\nu_{\sigma}$.

REMARK 3.2. Similarly, the quantized Steinhaus system associated to $\left(\Sigma, \mathbf{d}_{\Sigma}\right)$ is a collection $\mathcal{S}=\left\{\xi^{\sigma}: \Omega \rightarrow U\left(d_{\sigma}\right)\right\}_{\sigma \in \Sigma}$ of independent random unitary matrices, uniformly distributed on the unitary group $U\left(d_{\sigma}\right)$ equipped with its normalized Haar measure $\lambda_{\sigma}$. It is easy to check that both Rademacher and Steinhaus systems are u.b.q.o.s.'s with uniform bound $\mathrm{M}_{\mathcal{R}}=\mathrm{M}_{\mathcal{S}}=1$. Moreover, the notions of $\mathcal{R}$-type $p$ and $\mathcal{S}$-type $p$ are equivalent for $1 \leq p \leq 2$. Namely, the inequalities

$$
\frac{1}{2}\left\|\mathcal{F}_{\mathcal{R}}^{-1}(A)\right\|_{L_{B}^{q}(\Omega)} \leq\left\|\mathcal{F}_{\mathcal{S}}^{-1}(A)\right\|_{L_{B}^{q}(\Omega)} \leq 2\left\|\mathcal{F}_{\mathcal{R}}^{-1}(A)\right\|_{L_{B}^{q}(\Omega)}
$$

were proved in [10] for any Banach space $B$, any $A$ supported in any finite subset $\Sigma_{0}$ of $\Sigma$ and any $1 \leq q<\infty$. Hence, given an operator space $E$, we just need to take $B=S_{n}^{p^{\prime}}(E)$ for any $n \geq 1$ and $q=p^{\prime}$ to see this equivalence. Similar arguments are valid to show that the same equivalence holds between $\mathcal{R}$-cotype and $\mathcal{S}$-cotype. Moreover, the equivalence between both systems with respect to the strong Riesz cotype follows by duality. Therefore, although the results obtained will be valid for both systems, we shall work only with the quantized Rademacher system.

REMARK 3.3. Let $\mathrm{R}_{p}(E)$ be the closure in $L_{E}^{p}[0,1]$ of the subspace of linear combinations of the classical Rademacher functions $r_{1}, r_{2}, \ldots$ with $E$ valued coefficients. In particular, we shall write $\mathrm{R}_{p}$ for the closure in $L^{p}[0,1]$ of the subspace spanned by $r_{1}, r_{2}, \ldots$ The classical Khinchin-Kahane inequalities can be rephrased by saying that the norm of $\mathrm{R}_{p}(E)$, regarded as a Banach space, is equivalent to that of $\mathrm{R}_{q}(E)$ whenever $1 \leq p \neq q<\infty$. In particular we can put any exponent $1 \leq q<\infty$ in the defining inequality of Rademacher type $p$ (resp. cotype $p^{\prime}$ ) for (the underlying Banach space of) $E$,

$$
c_{1}\left(\sum_{k=1}^{n}\left\|e_{k}\right\|_{E}^{p^{\prime}}\right)^{1 / p^{\prime}} \leq\left\|\sum_{k=1}^{n} e_{k} r_{k}\right\|_{L_{E}^{q}[0,1]} \leq c_{2}\left(\sum_{k=1}^{n}\left\|e_{k}\right\|_{E}^{p}\right)^{1 / p} .
$$

On the other hand, $\mathrm{R}_{p}(E)$ has a natural operator space structure inherited from $L_{E}^{p}[0,1]$. It is a remarkable fact that the norm of $\mathrm{R}_{p}(E)$ is not completely equivalent to that of $\mathrm{R}_{q}(E)$. That is, the operator spaces $\mathrm{R}_{p}(E)$ and $\mathrm{R}_{q}(E)$ are isomorphic but not completely isomorphic. The proof of this fact is due to Pisier and it can be found in Chapter 8 of [13]. If we replace $r_{1}, r_{2}, \ldots$ by the entries of a quantized Rademacher system $\mathcal{R}$, then we obtain an operator space $\mathcal{R}_{p}(E)$ which is Banach isomorphic but not completely isomorphic to $\mathcal{R}_{q}(E)$ whenever $1 \leq p \neq q<\infty$. This equivalence of norms, which fails to be complete, follows from a version of the Khinchin-Kahane 
inequalities for $\mathcal{R}$ stated in [10]. Therefore, in contrast with (1), each choice of the exponent $1 \leq q<\infty$ in Definition 2.4 gives a different notion of Rademacher type and cotype. For instance, one could be tempted to take $q$ to be 2 whatever the value of $p$. In fact, this alternative definition becomes very useful in some other contexts which do not appear in this paper, such as the study of the notion of non-trivial Rademacher type. In any case, we have no risk in this paper to choose the wrong definition since we shall be mainly concerned with the quadratic case $p=2$.

Now we prove the extremality of the quantized Rademacher system with respect to Riesz type and cotype in the family of uniformly bounded quantized orthonormal systems. We shall need the following version, given in [10], of the classical contraction principle.

Lemma 3.4. Let $B$ be a Banach space, $\Sigma_{0} \subset \Sigma$ finite, $A^{\sigma} \in M_{d_{\sigma}} \otimes B$ and $D^{\sigma} \in M_{d_{\sigma}}$ for $\sigma \in \Sigma_{0}$. Then, for any $1 \leq q<\infty$,

$$
\left\|\sum_{\sigma \in \Sigma_{0}} d_{\sigma} \operatorname{tr}\left(A^{\sigma} \varrho^{\sigma} D^{\sigma}\right)\right\|_{L_{B}^{q}(\Omega)} \leq \sup _{\sigma \in \Sigma_{0}}\left\|D^{\sigma}\right\|_{S_{d_{\sigma}}^{\infty}}\left\|\sum_{\sigma \in \Sigma_{0}} d_{\sigma} \operatorname{tr}\left(A^{\sigma} \varrho^{\sigma}\right)\right\|_{L_{B}^{q}(\Omega)} .
$$

Proposition 3.5. Let $1 \leq p \leq 2$ and let $p^{\prime}$ denote its conjugate exponent. Then the following holds for any operator space $E$ and any u.b.q.o.s. $\Phi$ :

(i) If $E$ has $\Phi$-type $p$, then $E$ has $\mathcal{R}$-type $p$.

(ii) If $E$ has $\Phi$-cotype $p^{\prime}$, then $E$ has $\mathcal{R}$-cotype $p^{\prime}$.

Proof. The case $p=1$ is trivial, hence we assume that $E$ has $\Phi$-type $p$ for some $1<p \leq 2$. First we recall the completely isometric isomorphism

$$
S_{n}^{p^{\prime}}\left(L_{E}^{p^{\prime}}(\Omega)\right)=L_{S_{n}^{p^{\prime}}(E)}^{p^{\prime}}(\Omega) .
$$

On the other hand, by the orthonormality of $\Phi$ we have

$$
\int_{\Omega}\left|\varphi^{\sigma}\right|^{2} d \mu=I_{M_{d \sigma}}
$$

for all $\sigma \in \Sigma$. Hence, given $n \geq 1$ and $A_{i j} \in \mathcal{L}_{E}^{p}\left(\Sigma_{0}\right)$ for $1 \leq i, j \leq n$, we apply (2), (3), Jensen's inequality and the contraction principle stated in Lemma 3.4 to get

$$
\begin{aligned}
& \left\|\left(\sum_{\sigma \in \Sigma_{0}} d_{\sigma} \operatorname{tr}\left(A_{i j}^{\sigma} \varrho^{\sigma}\right)\right)\right\|_{S_{n}^{p^{\prime}}\left(L_{E}^{p^{\prime}}(\Omega)\right)} \\
& =\left[\int_{\Omega}\left\|\int_{\Omega}\left(\sum_{\sigma \in \Sigma_{0}} d_{\sigma} \operatorname{tr}\left[\varrho^{\sigma}\left(\omega_{1}\right)\left|\varphi^{\sigma}\left(\omega_{2}\right)\right|^{2} A_{i j}^{\sigma}\right]\right) d \mu\left(\omega_{2}\right)\right\|_{S_{n}^{p^{\prime}}(E)}^{p^{\prime}} d \mu\left(\omega_{1}\right)\right]^{1 / p^{\prime}} \\
& \leq\left[\iint_{\Omega}\left\|\left(\sum_{\sigma \in \Sigma_{0}} d_{\sigma} \operatorname{tr}\left[\varrho^{\sigma}\left(\omega_{1}\right) \varphi^{\sigma}\left(\omega_{2}\right)^{\star} \varphi^{\sigma}\left(\omega_{2}\right) A_{i j}^{\sigma}\right]\right)\right\|_{S_{n}^{p^{\prime}(E)}}^{p^{\prime}} d \mu\left(\omega_{1}\right) d \mu\left(\omega_{2}\right)\right]^{1 / p^{\prime}}
\end{aligned}
$$




$$
\begin{aligned}
& \leq \mathrm{M}_{\Phi}\left[\iint_{\Omega}\left\|\left(\sum_{\sigma \in \Sigma_{0}} d_{\sigma} \operatorname{tr}\left[\varrho^{\sigma}\left(\omega_{1}\right) \varphi^{\sigma}\left(\omega_{2}\right) A_{i j}^{\sigma}\right]\right)\right\|_{S_{n}^{p^{\prime}(E)}}^{p^{\prime}} d \mu\left(\omega_{2}\right) d \mu\left(\omega_{1}\right)\right]^{1 / p^{\prime}} \\
& \leq \mathrm{M}_{\Phi} \mathcal{K}_{1 p}(E, \Phi)\left[\int_{\Omega}\left\|\left(A_{i j}^{\sigma} \varrho^{\sigma}\left(\omega_{1}\right)\right)_{\sigma \in \Sigma_{0}}\right\|_{S_{n}^{p^{\prime}}\left(\mathcal{L}_{E}^{p}\left(\Sigma_{0}\right)\right)}^{p^{\prime}} d \mu\left(\omega_{1}\right)\right]^{1 / p^{\prime}} .
\end{aligned}
$$

Finally, by Lemma 1.7 of [13], it remains to see that

$$
\left[\int_{\Omega}\left\|\left(A_{i j}^{\sigma} \varrho^{\sigma}\left(\omega_{1}\right)\right)_{\sigma \in \Sigma_{0}}\right\|_{S_{n}^{p^{\prime}}\left(\mathcal{L}_{E}^{p}\left(\Sigma_{0}\right)\right)}^{p^{\prime}} d \mu\left(\omega_{1}\right)\right]^{1 / p^{\prime}}=\left\|\left(A_{i j}\right)\right\|_{S_{n}^{p^{\prime}}\left(\mathcal{L}_{E}^{p}\left(\Sigma_{0}\right)\right)} .
$$

To this end it is enough to check that the mapping $A \mapsto\left(A^{\sigma} \varrho^{\sigma}(\omega)\right)_{\sigma \in \Sigma_{0}}$ is a complete isometry from $\mathcal{L}_{E}^{p}\left(\Sigma_{0}\right)$ into itself. But this follows from the fact that $\varrho^{\sigma}(\omega) \in O\left(d_{\sigma}\right)$ for all $\sigma \in \Sigma$ and all $\omega \in \Omega$ (see Lemma 1.6 of [13]). This gives the estimate $\mathcal{K}_{1 p}(E, \mathcal{R}) \leq \mathrm{M}_{\Phi} \mathcal{K}_{1 p}(E, \Phi)$. Similar arguments give the relation $\mathcal{K}_{2 p^{\prime}}(E, \mathcal{R}) \leq \mathrm{M}_{\Phi} \mathcal{K}_{2 p^{\prime}}(E, \Phi)$.

REMARK 3.6. By duality, a similar result holds for the strong cotype.

The following is a classical result which characterizes, in terms of the convergence of some series of vector-valued random variables, Rademacher type (resp. cotype) 2 Banach spaces. The proof can be found in $[1$, Theorem 7.2 of Chapter 3].

Lemma 3.7. (i) The Banach space B has Rademacher type 2 if and only if there exists a sequence $\zeta_{1}, \zeta_{2}, \ldots$ of mean zero independent random variables in $L^{2}(\Omega)$ with $0<c_{1} \leq\left\|\zeta_{n}\right\|_{L^{2}(\Omega)} \leq c_{2}<\infty$ such that if $x_{1}, x_{2}, \ldots$ is any sequence in $B$, then

$$
\sum_{k=1}^{\infty}\left\|x_{k}\right\|_{B}^{2}<\infty \Rightarrow \sum_{k=1}^{n} x_{k} \zeta_{k} \text { converges a.s. }
$$

(ii) The Banach space $B$ has Rademacher cotype 2 if and only if there exists a sequence $\zeta_{1}, \zeta_{2}, \ldots$ of mean zero independent random variables in $L^{2}(\Omega)$ with $0<c_{1} \leq\left\|\zeta_{n}\right\|_{L^{2}(\Omega)} \leq c_{2}<\infty$ such that if $x_{1}, x_{2}, \ldots$ is any sequence in $B$, then

$$
\sum_{k=1}^{n} x_{k} \zeta_{k} \text { converges in } L^{2}(\Omega) \Rightarrow \sum_{k=1}^{\infty}\left\|x_{k}\right\|_{B}^{2}<\infty .
$$

LemMA 3.8. (i) Let $E$ be an operator space having $\mathcal{R}$-type 2 . Then the underlying Banach space has Rademacher type 2.

(ii) Let $E$ be an operator space having $\mathcal{R}$-cotype 2 . Then the underlying Banach space has Rademacher cotype 2.

Proof. Take a countable subset $\left\{\sigma_{k}: k \geq 1\right\}$ of $\Sigma$ and define the random variables $\zeta_{k}=\sqrt{d_{\sigma_{k}}} \varrho_{11}^{\sigma_{k}}$ for $k \geq 1$. The sequence $\zeta_{1}, \zeta_{2}, \ldots$ is orthonormal in $L^{2}(\Omega)$ and is made up of mean zero independent random variables. 
Moreover, if we take any square-summable sequence $x_{1}, x_{2}, \ldots$ in $E$ and $A^{k} \in M_{d_{\sigma_{k}}} \otimes E$ is defined by $A_{i j}^{k}=\delta_{i 1} \delta_{j 1} d_{\sigma_{k}}^{-1 / 2} x_{k}$, then

$$
\begin{aligned}
\left\|\sum_{k=m_{1}+1}^{m_{2}} x_{k} \zeta_{k}\right\|_{L_{E}^{2}(\Omega)} & =\left\|\sum_{k=m_{1}+1}^{m_{2}} d_{\sigma_{k}} \operatorname{tr}\left(A^{k} \varrho^{\sigma_{k}}\right)\right\|_{L_{E}^{2}(\Omega)} \\
& \leq \mathcal{K}_{12}(E, \mathcal{R})\left(\sum_{k=m_{1}+1}^{m_{2}} d_{\sigma_{k}}\left\|A^{k}\right\|_{S_{d_{\sigma_{k}}}^{2}(E)}^{2}\right)^{1 / 2} \\
& =\mathcal{K}_{12}(E, \mathcal{R})\left(\sum_{k=m_{1}+1}^{m_{2}}\left\|x_{k}\right\|_{E}^{2}\right)^{1 / 2}
\end{aligned}
$$

Similarly, we get

$$
\left(\sum_{k=m_{1}+1}^{m_{2}}\left\|x_{k}\right\|_{E}^{2}\right)^{1 / 2} \leq \mathcal{K}_{22}(E, \mathcal{R})\left\|\sum_{k=m_{1}+1}^{m_{2}} x_{k} \zeta_{k}\right\|_{L_{E}^{2}(\Omega)}
$$

That is, we have proved that

$$
\sum_{k=1}^{\infty}\left\|x_{k}\right\|_{E}^{2}<\infty \Leftrightarrow \sum_{k=1}^{n} x_{k} \zeta_{k} \text { converges in } L_{E}^{2}(\Omega) .
$$

But convergence in $L_{E}^{2}(\Omega)$ implies a.s. convergence for this kind of series (see Theorem 2.10 in Chapter 3 of [1]). The proof is concluded by applying Lemma 3.7.

REMARK 3.9. By duality, a similar result holds for the strong cotype.

In this section we explore Kwapień's theorem in the present context, that is, completely isomorphic characterizations of Pisier's OH Hilbertian operator spaces by means of quantized orthonormal systems. Roughly speaking, an $\mathrm{OH}$ operator space is the only possible quantization on a Hilbert space such that the canonical identification between the resulting operator space and its antidual is a complete isometry; see [12] for a complete study of these spaces. In other words, the $\mathrm{OH}$ operator spaces are the natural substitutes for classical Hilbert spaces in the category of operator spaces.

ThEOREM 3.10. Let $\Phi$ be any u.b.q.o.s. associated to the parameters $\left(\Sigma, \mathbf{d}_{\Sigma}\right)$. Let $E$ be an operator space. Then the following are equivalent:

(i) $E$ is completely isomorphic to some $\mathrm{OH}$ Hilbertian operator space.

(ii) E has $\Phi$-type and $\Phi$-cotype 2.

Proof. We begin by showing (i) $\Rightarrow($ ii). Assume that $E$ is completely isomorphic to $\mathrm{OH}(I)$ for some index set $I$. We invoke the general results stated in Section 2 to write $\mathcal{K}_{12}(E, \Phi) \leq d_{\mathrm{cb}}(E, \mathrm{OH}(I)) \mathcal{K}_{12}(\mathrm{OH}(I), \Phi)$. But $\mathrm{OH}(I)$ is completely isometric to $l^{2}(I)$ and it is not difficult to check that 
$\mathcal{K}_{12}\left(l^{2}(I), \Phi\right)=1$. This implies that $E$ has $\Phi$-type 2 . Similar arguments show that $E$ also has $\Phi$-cotype 2 .

Now we prove (ii) $\Rightarrow$ (i). Suppose that $E$ has $\Phi$-type and $\Phi$-cotype 2 . By Proposition 3.5 we can replace $\Phi$ by the quantized Rademacher system $\mathcal{R}$ with parameters $\left(\Sigma, \mathbf{d}_{\Sigma}\right)$. We know that $S^{2}(E)$ also has $\mathcal{R}$-type and $\mathcal{R}$-cotype 2 (see again the general results of Section 2 ). Now, Lemma 3.8 shows that (the underlying Banach space of) $S^{2}(E)$ has Rademacher type and cotype 2. In particular, $S^{2}(E)$ is isomorphic to some Hilbert space. By Kwapień's original theorem, this geometric condition on $S^{2}(E)$ is equivalent to the existence of a constant $c$ such that $\left\|T \otimes I_{S^{2}(E)}\right\|_{\mathcal{B}\left(l_{n}^{2}\left(S^{2}(E)\right), l_{n}^{2}\left(S^{2}(E)\right)\right)} \leq$ $c\|T\|_{\mathcal{B}\left(l_{n}^{2}, l_{n}^{2}\right)}$ for any linear mapping $T: l_{n}^{2} \rightarrow l_{n}^{2}$ and any $n \geq 1$ (see [6]). On the other hand, the Fubini complete isometry $l_{n}^{2}\left(S^{2}(E)\right) \simeq S^{2}\left(l_{n}^{2}(E)\right)$ given in [13] allows us to write the last inequality as $\left\|T \otimes I_{E}\right\|_{\operatorname{cb}\left(l_{n}^{2}(E), l_{n}^{2}(E)\right)} \leq$ $c\|T\|_{\mathcal{B}\left(l_{n}^{2}, l_{n}^{2}\right)}$. Finally, Pisier proved that this condition is equivalent to (i) (see Theorem 6.11 of [13]).

4. Complete quantized orthonormal systems. In this section we extend the operator space version of Kwapień's theorem to complete quantized orthonormal systems, uniformly bounded or not. We begin by recalling that since $(\Omega, \mathcal{M}, \mu)$ has no atoms, we can define a family of dyadic sets $\mathrm{D}_{j}^{k}$ on $\Omega$, where $1 \leq j \leq 2^{k}$ and $k \geq 1$, satisfying the following conditions:

- $\mathrm{D}_{j}^{k}=\mathrm{D}_{2 j-1}^{k+1} \cup \mathrm{D}_{2 j}^{k+1}$ for all $k \geq 1$ and all $1 \leq j \leq 2^{k}$.

- $\Omega$ is the disjoint union of $\mathrm{D}_{j}^{k}$ for any fixed $k \geq 1$ and all $1 \leq j \leq 2^{k}$.

- The sets $\mathrm{D}_{j}^{k}$ are $\mu$-measurable and $\mu\left(\mathrm{D}_{j}^{k}\right)=2^{-k}$.

Then, if $1_{\Lambda}$ stands for the characteristic function of a measurable set $\Lambda \subset \Omega$, we define the system $\Delta$ on $L^{2}(\Omega)$ to consist of the functions

$$
\delta_{k}=\sum_{j=1}^{2^{k}}(-1)^{j+1} 1_{\mathrm{D}_{j}^{k}}
$$

Lemma 4.1. Let $\Psi=\left\{\psi^{\sigma}: \Omega \rightarrow M_{d_{\sigma}}\right\}_{\sigma \in \Sigma}$ be a complete quantized orthonormal system. Let $\left\{\varepsilon_{n}: n \geq 1\right\}$ be any sequence of positive numbers. Then there exists a sequence $f_{1}, f_{2}, \ldots$ of $\Psi$-polynomials and an increasing subsequence $k_{1}, k_{2} \ldots$ of positive integers satisfying:

(i) $\mathcal{F}_{\Psi}\left(f_{1}\right), \mathcal{F}_{\Psi}\left(f_{2}\right)$, . . have pairwise disjoint supports on $\Sigma$.

(ii) $\left\|f_{n}-\delta_{k_{n}}\right\|_{L^{2}(\Omega)}<\varepsilon_{n}$.

Proof. Let $\sigma \in \Sigma$ and fix $1 \leq i, j \leq d_{\sigma}$. Since $\Delta$ is orthonormal in $L^{2}(\Omega)$, the Bessel inequality provides the estimate

$$
\sum_{k=1}^{\infty}\left|\mathcal{F}_{\Psi}\left(\delta_{k}\right)_{i j}^{\sigma}\right|^{2}=\sum_{k=1}^{\infty}\left|\int_{\Omega} \delta_{k}(\omega) \overline{\psi_{j i}^{\sigma}(\omega)} d \mu(\omega)\right|^{2} \leq\left\|\psi_{j i}^{\sigma}\right\|_{L^{2}(\Omega)}^{2}=\frac{1}{d_{\sigma}}<\infty .
$$


In particular, for all $\epsilon>0$ and for all finite subsets $\Sigma_{0} \subset \Sigma$ there exists a positive integer $m\left(\Sigma_{0}, \epsilon\right)$ such that for all $k \geq m\left(\Sigma_{0}, \epsilon\right)$ we have

$$
\sum_{\sigma \in \Sigma_{0}} d_{\sigma} \sum_{i, j=1}^{d_{\sigma}}\left|\mathcal{F}_{\Psi}\left(\delta_{k}\right)_{i j}^{\sigma}\right|^{2}<\epsilon
$$

On the other hand, let $\Psi_{0}$ be the space of $\Psi$-polynomials. That is, $\Psi_{0}$ is the span of the entries $\psi_{i j}^{\sigma}$ where $1 \leq i, j \leq d_{\sigma}$ and $\sigma \in \Sigma$. Then we construct the functions $f_{1}, f_{2}, \ldots$ as follows. Let $f_{1} \in \Psi_{0}$ be such that $\left\|f_{1}-\delta_{1}\right\|_{L^{2}(\Omega)}<\varepsilon_{1}$. For $n>1$, let $\epsilon_{n}=\varepsilon_{n} / 3$ and let

$$
\Sigma_{n}=\bigcup_{k=1}^{n-1} \operatorname{supp}\left(\mathcal{F}_{\Psi}\left(f_{k}\right)\right) \subset \Sigma .
$$

If $k_{n}=m\left(\Sigma_{n}, \epsilon_{n}\right)$ we take $g_{n}$ to be any function in $\Psi_{0}$ satisfying $\left\|g_{n}-\delta_{k_{n}}\right\|_{L^{2}(\Omega)}<\epsilon_{n}$. Then we define

$$
f_{n}=g_{n}-\sum_{\sigma \in \Sigma_{n}} d_{\sigma} \operatorname{tr}\left(\mathcal{F}_{\Psi}\left(g_{n}\right)^{\sigma} \psi^{\sigma}\right)
$$

The verification that the sequence $f_{1}, f_{2}, \ldots$ has the required properties is left to the reader.

THEOREM 4.2. Let $\Psi$ be any complete quantized orthonormal system with parameters $\left(\Sigma, \mathbf{d}_{\Sigma}\right)$. Let $E$ be an operator space. The following are equivalent:

(i) $E$ is completely isomorphic to some $\mathrm{OH}$ Hilbertian operator space.

(ii) E has $\Psi$-type and $\Psi$-cotype 2.

Proof. The arguments used in Theorem 3.10 to prove (i) $\Rightarrow$ (ii) are also valid here. Let us prove that (ii) $\Rightarrow(\mathrm{i})$. First we recall that, by Lemma 4.1, there exists a sequence $f_{1}, f_{2}, \ldots$ of $\Psi$-polynomials

$$
f_{n}=\sum_{\sigma \in \Sigma_{n}} \sum_{1 \leq i, j \leq d_{\sigma}} \alpha_{i j}^{\sigma} \psi_{i j}^{\sigma}
$$

where $\alpha_{i j}^{\sigma} \in \mathbb{C}, \Sigma_{n}$ is some finite subset of $\Sigma$ and

$$
\Sigma_{n_{1}} \cap \Sigma_{n_{2}}=\emptyset \quad \text { whenever } n_{1} \neq n_{2}, \quad\left\|f_{n}-\delta_{k_{n}}\right\|_{L^{2}(\Omega)}<2^{-n} .
$$

Now, if $E$ has $\Psi$-type and $\Psi$-cotype 2 then the same holds for $F=S^{2}(E)$. In particular, for any family $\left\{x_{1}, \ldots, x_{n}\right\}$ in $F$, we have

$$
\left\|\sum_{n=1}^{m} x_{n} \delta_{k_{n}}\right\|_{L_{F}^{2}(\Omega)} \leq\left\|\sum_{n=1}^{m} x_{n}\left(\delta_{k_{n}}-f_{n}\right)\right\|_{L_{F}^{2}(\Omega)}+\left\|\sum_{n=1}^{m} x_{n} f_{n}\right\|_{L_{F}^{2}(\Omega)}=\mathrm{A}+\mathrm{B} .
$$


By Hölder's inequality we get

$$
\mathrm{A} \leq\left(\sum_{n=1}^{m}\left\|f_{n}-\delta_{k_{n}}\right\|_{L^{2}(\Omega)}^{2}\right)^{1 / 2}\left(\sum_{n=1}^{m}\left\|x_{n}\right\|_{F}^{2}\right)^{1 / 2} \leq \frac{1}{\sqrt{3}}\left(\sum_{n=1}^{m}\left\|x_{n}\right\|_{F}^{2}\right)^{1 / 2} .
$$

To estimate B, we write

$$
\begin{aligned}
\mathrm{B} & =\left\|\sum_{n=1}^{m} x_{n} \sum_{\sigma \in \Sigma_{n}} \sum_{1 \leq i, j \leq d_{\sigma}} \alpha_{i j}^{\sigma} \psi_{i j}^{\sigma}\right\|_{L_{F}^{2}(\Omega)} \\
& =\left\|\sum_{n=1}^{m} \sum_{\sigma \in \Sigma_{n}} d_{\sigma} \operatorname{tr}\left[\left(\mathcal{F}_{\Psi}\left(f_{n}\right)^{\sigma} \otimes x_{n}\right) \psi^{\sigma}\right]\right\|_{L_{F}^{2}(\Omega)} \\
& \leq \mathcal{K}_{12}(E, \Psi)\left(\sum_{n=1}^{m}\left\|x_{n}\right\|_{F}^{2} \sum_{\sigma \in \Sigma_{n}} d_{\sigma}\left\|\mathcal{F}_{\Psi}\left(f_{n}\right)^{\sigma}\right\|_{S_{d_{\sigma}}^{2}}^{2}\right)^{1 / 2} \\
& =\mathcal{K}_{12}(E, \Psi)\left(\sum_{n=1}^{m}\left\|x_{n}\right\|_{F}^{2}\left\|f_{n}\right\|_{L^{2}(\Omega)}^{2}\right)^{1 / 2} \\
& \leq 2 \mathcal{K}_{12}(E, \Psi)\left(\sum_{n=1}^{m}\left\|x_{n}\right\|_{F}^{2}\right)^{1 / 2} .
\end{aligned}
$$

That is, if $\Delta^{\prime}$ stands for the system in $L^{2}(\Omega)$ defined by the functions $\delta_{k_{1}}, \delta_{k_{2}}, \ldots$, then we have shown that $F$ has $\Delta^{\prime}$-type 2 in the sense of [3]. But this is equivalent to saying that $E$ has $\Delta^{\prime}$-type 2 in the sense of Definition 2.4. Similar arguments show that $E$ also has $\Delta^{\prime}$-cotype 2. Then the proof is concluded by applying Theorem 3.10.

REMARK 4.3. The analog of Kwapień's argument given in [6] does not work for this result. Namely, if $\mathcal{R}$ denotes the quantized Rademacher system with parameters $\left(\Sigma, \mathbf{d}_{\Sigma}\right)$, the idea is to use the completeness of $\Psi$ to construct a sequence $f^{\sigma_{1}}, f^{\sigma_{2}}, \ldots$ of matrix-valued $\Psi$-polynomials with non-overlapping ranges of frequencies and such that

$$
\int_{\Omega}\left\|\varrho^{\sigma_{n}}-f^{\sigma_{n}}\right\|_{S_{d_{\sigma_{n}}}^{2}}^{2} d \mu<\varepsilon_{n} \quad \text { with } \varepsilon_{1}, \varepsilon_{2}, \ldots \text { small enough. }
$$

Such a sequence exists and its construction is similar to the one provided in Lemma 4.1. If $\mathcal{R}^{\prime}$ denotes the subsystem of $\mathcal{R}$ defined by the functions $\varrho^{\sigma_{1}}, \varrho^{\sigma_{2}}, \ldots$, the next step is to show that $\Psi$-type 2 implies $\mathcal{R}^{\prime}$-type 2 and the same for the cotype. Here the proof fails. However, it can be checked that it works in the following cases:

- $\Psi$-type $2 \Rightarrow \mathcal{R}^{\prime}$-type 2 if $\mathbf{d}_{\Sigma}$ is bounded.

- $\Psi$-cotype $2 \Rightarrow \mathcal{R}^{\prime}$-cotype 2 if $d_{\sigma}=1$ for all $\sigma \in \Sigma$.

5. The probabilistic approach. In this section we introduce the quantization of the classical Gauss system and analyze its important role in the 
operator space version of Kwapień's theorem. First we outline a simple proof of Kwapień's theorem for this system and then we give an alternative proof following Kwapień's approach in [6] conveniently adapted to our setting. The reason for this approach will be clear in Corollary 5.7.

Definition 5.1. Let $\left\{\gamma_{i j}^{\sigma}: \Omega \rightarrow \mathbb{R}, 1 \leq i, j \leq d_{\sigma}\right\}_{\sigma \in \Sigma}$ be a family of independent real gaussian random variables with mean zero and variance 1 . Then the collection $\mathcal{G}=\left\{\gamma^{\sigma}: \Omega \rightarrow M_{d_{\sigma}}\right\}_{\sigma \in \Sigma}$, where $\gamma^{\sigma}$ stands for the random matrix

$$
\gamma^{\sigma}=\frac{1}{\sqrt{d_{\sigma}}}\left(\gamma_{i j}^{\sigma}\right)
$$

defines the quantized gaussian system associated to $\left(\Sigma, \mathbf{d}_{\Sigma}\right)$.

REMARK 5.2. Analogously, considering a priori complex gaussian random variables, we get the quantized complex gaussian system associated to $\left(\Sigma, \mathbf{d}_{\Sigma}\right)$.

This quantized system satisfies orthonormality but fails to be uniformly bounded or complete. So none of the previous results seems to be valid for the quantized gaussian system. However, it is not difficult to check that Lemma 3.8 remains valid when we replace the quantized Rademacher system $\mathcal{R}$ by the quantized gaussian system $\mathcal{G}$. In particular, the proof of Theorem 3.10 also holds for $\mathcal{G}$.

We now give an alternative approach to this result. Let $\widetilde{\Omega}$ be the probability space formed by the product of infinitely many copies of $\Omega$ :

$$
\widetilde{\Omega}=\prod_{k=1}^{\infty} \Omega_{k} \quad \text { and } \quad \widetilde{\mu}=\prod_{k=1}^{\infty} \mu_{k}
$$

with $\Omega_{k}=\Omega$ and $\mu_{k}=\mu$ for all $k \geq 1$. The random matrix $\varrho^{\sigma, k}: \widetilde{\Omega} \rightarrow O\left(d_{\sigma}\right)$ is defined as a copy of $\varrho^{\sigma}$, the $\sigma$ th Rademacher function, depending only on the $k$ th coordinate. Also, for each positive integer $m$, we define

$$
\varrho^{\sigma}(m): \widetilde{\Omega} \rightarrow M_{d_{\sigma}}, \quad \varrho^{\sigma}(m)=\frac{1}{\sqrt{m}} \sum_{k=1}^{m} \varrho^{\sigma, k} .
$$

Finally, we construct a quantized gaussian system $\left\{\widetilde{\gamma}^{\sigma}: \widetilde{\Omega} \rightarrow M_{d_{\sigma}}\right\}_{\sigma \in \Sigma}$ on $\widetilde{\Omega}$ associated to the parameters $\left(\Sigma, \mathbf{d}_{\Sigma}\right)$. We state a slight modification of the central limit theorem in type 2 spaces (see [1] for the classical statement of that result). It is nothing but an analog, for Banach-valued random variables, of Lemma 2.1 in [6]. Fix a finite subset $\Sigma_{0}=\left\{\sigma_{1}, \ldots, \sigma_{n}\right\}$ of $\Sigma$.

Proposition 5.3. Let $h: S_{d_{\sigma_{1}}}^{2} \times \ldots \times S_{d_{\sigma_{n}}}^{2} \rightarrow \mathbb{R}$ be a continuous function such that 


$$
h\left(D^{\sigma_{1}}, \ldots, D^{\sigma_{n}}\right) e^{-\sum_{j=1}^{n}\left\|D^{\sigma_{j}}\right\|} \rightarrow 0 \quad \text { as } \quad \sum_{j=1}^{n}\left\|D^{\sigma_{j}}\right\|_{S_{d_{\sigma_{j}}}^{2}} \rightarrow \infty .
$$

Then

$$
\lim _{m \rightarrow \infty} \int_{\widetilde{\Omega}} h\left(\varrho^{\sigma_{1}}(m), \ldots, \varrho^{\sigma_{n}}(m)\right) d \widetilde{\mu}=\int_{\widetilde{\Omega}} h\left(\widetilde{\gamma}^{\sigma_{1}}, \ldots, \widetilde{\gamma}^{\sigma_{n}}\right) d \widetilde{\mu} .
$$

Sketch of the proof. By using the orthonormality relations for quantized Rademacher and gaussian systems, one easily shows that the distribution of $\widetilde{\gamma}^{\sigma}$ is a centered cylindrical gaussian measure with the same covariance as that of $\varrho^{\sigma, k}$ for all $k \geq 1$. Hence, by the central limit theorem in type 2 spaces, the joint distribution of $\left(\varrho^{\sigma_{1}}(m), \ldots, \varrho^{\sigma_{n}}(m)\right)$ converges weakly to the joint distribution of $\left(\widetilde{\gamma}^{\sigma_{1}}, \ldots, \widetilde{\gamma}^{\sigma_{n}}\right)$. Now, if we write $S_{\Sigma_{0}}^{2}=S_{d_{\sigma_{1}}}^{2} \times \ldots \times S_{d_{\sigma_{n}}}^{2}$, we define the Banach space $B$ of all continuous functions $h: S_{\Sigma_{0}}^{2} \rightarrow \mathbb{R}$ satisfying (4) and with the norm given by

$$
\|h\|_{B}=\sup \left\{\left|h\left(D^{\sigma_{1}}, \ldots, D^{\sigma_{n}}\right)\right| e^{-\sum_{j=1}^{n}\left\|D^{\sigma_{j}}\right\|}:\left(D^{\sigma_{1}}, \ldots, D^{\sigma_{n}}\right) \in S_{\Sigma_{0}}^{2}\right\} .
$$

We also define the following functionals on $B$ :

$$
T(h)=\int_{\widetilde{\Omega}} h\left(\widetilde{\gamma}^{\sigma_{1}}, \ldots, \widetilde{\gamma}^{\sigma_{n}}\right) d \widetilde{\mu}, \quad T_{m}(h)=\int_{\widetilde{\Omega}} h\left(\varrho^{\sigma_{1}}(m), \ldots, \varrho^{\sigma_{n}}(m)\right) d \widetilde{\mu} .
$$

Following the argument given in Lemma 2.1 of [6], it suffices to check that $T$ and $T_{m}$ are well defined and that $\sup \left\|T_{m}\right\|<\infty$. Now, $T_{m}$ is well defined since $h\left(\varrho^{\sigma_{1}}(m), \ldots, \varrho^{\sigma_{n}}(m)\right)$ is a bounded function. On the other hand,

$$
\begin{aligned}
|T(h)| & \leq\|h\|_{B} \prod_{j=1}^{n} \int_{S_{d_{\sigma_{j}}}^{2}} \exp \left\|D^{\sigma_{j}}\right\|_{S_{d_{\sigma_{j}}}^{2}} d \mu_{\widetilde{\gamma}^{\sigma_{j}}}\left(D^{\sigma_{j}}\right) \\
& \leq\|h\|_{B} \prod_{j=1}^{n} \int_{\widetilde{\Omega}} \prod_{1 \leq i_{1}, i_{2} \leq d_{\sigma_{j}}} \exp \left|\frac{\widetilde{\gamma}_{i_{1} i_{2}}^{\sigma_{j}}(\widetilde{\omega})}{\sqrt{d_{\sigma_{j}}}}\right| d \widetilde{\mu}(\widetilde{\omega}) \\
& \leq\|h\|_{B} \prod_{j=1}^{n} \prod_{1 \leq i_{1}, i_{2} \leq d_{\sigma_{j}}}\left(\int_{\widetilde{\Omega}} \exp d_{\sigma_{j}}^{2}\left|\frac{\widetilde{\gamma}_{i_{1} i_{2}}^{\sigma_{j}}(\widetilde{\omega})}{\sqrt{d_{\sigma_{j}}}}\right| d \widetilde{\mu}(\widetilde{\omega})\right)^{1 / d_{\sigma_{j}}^{2}} \\
& =\|h\|_{B} \prod_{j=1}^{n} \prod_{1 \leq i_{1}, i_{2} \leq d_{\sigma_{j}}}\left(\frac{1}{\sqrt{2 \pi}} \int_{\mathbb{R}} \exp \left|d_{\sigma_{j}}^{3 / 2} s\right| \exp \left(-s^{2} / 2\right) d s\right)^{1 / d_{\sigma_{j}}^{2}}
\end{aligned}
$$

where we have applied the obvious inequality $\|D\|_{S_{n}^{2}} \leq \sum_{i j}\left|D_{i j}\right|$ and the generalized Hölder inequality. Therefore $T$ is well defined. Similar arguments give the uniform boundedness of $\left\|T_{m}\right\|$.

To prove the Kwapien theorem for the quantized gaussian system we need a couple of lemmas. Let $D_{1}, D_{2}$ be orthogonal $d_{\sigma} \times d_{\sigma}$ matrices. Then 
$D_{1} \gamma^{\sigma} D_{2}$ and $\gamma^{\sigma}$ have the same distribution. The next result can be found in [10], it follows from this "sign invariance" and the contraction principle stated above.

Lemma 5.4. Let B be a Banach space. There exists a positive constant $c$ such that for any finite subset $\Sigma_{0}$ of $\Sigma$, we have

$$
\int_{\Omega}\left\|\sum_{\sigma \in \Sigma_{0}} d_{\sigma} \operatorname{tr}\left(A^{\sigma} \varrho^{\sigma}\right)\right\|_{B}^{2} d \mu \leq c \int_{\Omega}\left\|\sum_{\sigma \in \Sigma_{0}} d_{\sigma} \operatorname{tr}\left(A^{\sigma} \gamma^{\sigma}\right)\right\|_{B}^{2} d \mu .
$$

The following result is a completely isomorphic characterization of $\mathrm{OH}$ operator spaces given by Pisier in [13]. It can be regarded as the version for operator spaces of a previous isomorphic characterization of Hilbert spaces given by Kwapień (see (iv) of Proposition 3.1 in [6]).

Lemma 5.5. Let $E$ be an operator space. Then $E$ is completely isomorphic to some $\mathrm{OH}$ Hilbertian operator space if and only if there exists a positive constant $c$ such that for any $n \geq 1$ and any linear mapping $T: S_{n}^{2} \rightarrow S_{n}^{2}$, we have

$$
\left\|T \otimes I_{E}\right\|_{\mathcal{B}\left(S_{n}^{2}(E), S_{n}^{2}(E)\right)} \leq c\|T\|_{\mathcal{B}\left(S_{n}^{2}, S_{n}^{2}\right)} .
$$

In the next result we assume that the gaussian system we work with takes values in arbitrarily large matrices. We need to require this in view of our method of proof. Although, as we have seen, this requirement is not necessary, it will become very natural in Corollary 5.7.

THEOREM 5.6. Let $\mathcal{G}$ be the gaussian system with parameters $\left(\Sigma, \mathbf{d}_{\Sigma}\right)$. Assume that $\mathbf{d}_{\Sigma}$ is unbounded. Then the following are equivalent:

(i) $E$ is completely isomorphic to some $\mathrm{OH}$ Hilbertian operator space.

(ii) $E$ has $\mathcal{G}$-type and $\mathcal{G}$-cotype 2 .

Proof. To prove that (i) $\Rightarrow$ (ii), assume that $E$ is completely isomorphic to some $\mathrm{OH}(I)$. If $\mathcal{R}$ denotes the quantized Rademacher system with parameters $\left(\Sigma, \mathbf{d}_{\Sigma}\right)$, then we know by Theorem 3.10 that $E$ has $\mathcal{R}$-type and $\mathcal{R}$-cotype 2 . But then Lemma 5.4 shows that $E$ has $\mathcal{G}$-cotype 2 . Let us prove that $E$ also has $\mathcal{G}$-type 2 . Recall that any Banach space $B$ with Rademacher type 2 satisfies the inequality

$$
\int_{\Omega}\left\|\sum_{k=1}^{n} \phi_{k}\right\|_{B}^{2} d \mu \leq c \sum_{k=1}^{n} \int_{\Omega}\left\|\phi_{k}\right\|_{B}^{2} d \mu
$$

for some universal constant $c$ and any family $\phi_{1}, \ldots, \phi_{n}$ of mean zero independent $B$-valued random variables in $L^{2}(\Omega)$. In particular, since (by Lemma 3.8) the underlying Banach space of $S_{n}^{2}(E)$ has Rademacher type 2 for any $n \geq 1$, we have

$$
\left\|\sum_{\sigma \in \Sigma_{0}} d_{\sigma} \operatorname{tr}\left(A^{\sigma} \varrho^{\sigma}(m)\right)\right\|_{S_{n}^{2}\left(L_{E}^{2}(\widetilde{\Omega})\right)}=\left\|\sum_{k=1}^{m} \sum_{\sigma \in \Sigma_{0}} d_{\sigma} \operatorname{tr}\left[\frac{A^{\sigma} \varrho^{\sigma, k}}{\sqrt{m}}\right]\right\|_{L_{S_{n}^{2}(E)}^{2}(\widetilde{\Omega})}
$$




$$
\begin{aligned}
& \leq c\left(\sum_{k=1}^{m}\left\|\sum_{\sigma \in \Sigma_{0}} d_{\sigma} \operatorname{tr}\left[\frac{A^{\sigma} \varrho^{\sigma, k}}{\sqrt{m}}\right]\right\|_{L_{S_{n}^{2}(E)}^{2}\left(\Omega_{k}\right)}^{2}\right)^{1 / 2} \\
& \leq c \mathcal{K}_{12}(E, \mathcal{R})\left(\sum_{\sigma \in \Sigma_{0}} d_{\sigma}\left\|A^{\sigma}\right\|_{S_{d_{\sigma} n}^{2}(E)}^{2}\right)^{1 / 2} .
\end{aligned}
$$

On the other hand, let $h\left(D^{\sigma_{1}}, \ldots, D^{\sigma_{n}}\right)=\left\|\sum_{\sigma \in \Sigma_{0}} d_{\sigma} \operatorname{tr}\left(A^{\sigma} D^{\sigma}\right)\right\|_{S_{n}^{2}(E)}^{2}$. Let us show that $h$ satisfies hypothesis (4) of Proposition 5.3. First we recall that

$$
\begin{aligned}
\left\|\operatorname{tr}\left(A^{\sigma} D^{\sigma}\right)\right\|_{S_{n}^{2}(E)} & =\sup _{\|T\|_{\left.S_{n}^{(} E^{\star}\right)}^{2} \leq 1} \operatorname{tr}\left(A^{\sigma}\left(D^{\sigma} \otimes T\right)\right) \\
& \leq \sup _{\|T\|_{S_{n}^{2}\left(E^{\star}\right)} \leq 1}\left\|A^{\sigma}\right\|_{S_{d_{\sigma} n}^{2}(E)}\left\|D^{\sigma} \otimes T\right\|_{S_{d_{\sigma} n}^{2}\left(E^{\star}\right)} \\
& =\left\|A^{\sigma}\right\|_{S_{d_{\sigma} n}^{2}(E)}\left\|D^{\sigma}\right\|_{S_{d_{\sigma}}^{2}}
\end{aligned}
$$

Hence we get

$$
\begin{aligned}
h\left(D^{\sigma_{1}}, \ldots, D^{\sigma_{n}}\right) & \leq\left(\sum_{\sigma \in \Sigma_{0}} d_{\sigma}\left\|A^{\sigma}\right\|_{S_{d_{\sigma} n}^{2}(E)}\left\|D^{\sigma}\right\|_{S_{d_{\sigma}}^{2}}\right)^{2} \\
& \leq \max _{\sigma \in \Sigma_{0}} d_{\sigma}^{2}\left\|A^{\sigma}\right\|_{S_{d_{\sigma} n}^{2}(E)}^{2}\left(\sum_{\sigma \in \Sigma_{0}}\left\|D^{\sigma}\right\|_{S_{d_{\sigma}}^{2}}\right)^{2}
\end{aligned}
$$

and so $h$ satisfies (4). In particular, we apply Proposition 5.3 to obtain

$$
\begin{aligned}
\left\|\sum_{\sigma \in \Sigma_{0}} d_{\sigma} \operatorname{tr}\left(A^{\sigma} \gamma^{\sigma}\right)\right\|_{S_{n}^{2}\left(L_{E}^{2}(\Omega)\right)} & =\left\|\sum_{\sigma \in \Sigma_{0}} d_{\sigma} \operatorname{tr}\left(A^{\sigma} \widetilde{\gamma}^{\sigma}\right)\right\|_{S_{n}^{2}\left(L_{E}^{2}(\widetilde{\Omega})\right)} \\
& =\lim _{m \rightarrow \infty}\left\|\sum_{\sigma \in \Sigma_{0}} d_{\sigma} \operatorname{tr}\left(A^{\sigma} \varrho^{\sigma}(m)\right)\right\|_{S_{n}^{2}\left(L_{E}^{2}(\widetilde{\Omega})\right)} \\
& \leq c \mathcal{K}_{12}(E, \mathcal{R})\left(\sum_{\sigma \in \Sigma_{0}} d_{\sigma}\left\|A^{\sigma}\right\|_{S_{d_{\sigma} n}^{2}(E)}^{2}\right)^{1 / 2}
\end{aligned}
$$

Therefore, by Lemma 1.7 of [13], we conclude that $E$ has $\mathcal{G}$-type 2 and the proof of $(\mathrm{i}) \Rightarrow$ (ii) is complete.

Now we show (ii) $\Rightarrow$ (i). By the unboundedness of $\mathbf{d}_{\Sigma}$ and Lemma 5.5 it suffices to see that there exists a positive constant $c$ such that, for any $\sigma \in \Sigma$ and any linear mapping $T: S_{d_{\sigma}}^{2} \rightarrow S_{d_{\sigma}}^{2}$, we have

$$
\left\|T \otimes I_{E}\right\|_{\mathcal{B}\left(S_{d_{\sigma}}^{2}(E), S_{d_{\sigma}}^{2}(E)\right)} \leq c\|T\|_{\mathcal{B}\left(S_{d_{\sigma}}^{2}, S_{d_{\sigma}}^{2}\right)} .
$$

By homogeneity it is enough to prove (5) for $T$ in the unit ball $B_{\sigma}$ of $\mathcal{B}\left(S_{d_{\sigma}}^{2}, S_{d_{\sigma}}^{2}\right)$. But $\mathrm{B}_{\sigma}$ is a compact, convex set and thus every element of $\mathrm{B}_{\sigma}$ is a convex linear combination of unitary operators, the extreme points of $\mathrm{B}_{\sigma}$. Therefore, it suffices to check (5) for $T$ unitary. Let $A \in S_{d_{\sigma}}^{2}(E)$ and $T: S_{d_{\sigma}}^{2} \rightarrow S_{d_{\sigma}}^{2}$ unitary. Then 


$$
\begin{aligned}
\left\|T \otimes I_{E}(A)\right\|_{S_{d_{\sigma}}^{2}(E)} & \leq d_{\sigma}^{-1 / 2} \mathcal{K}_{22}(E, \mathcal{G})\left\|d_{\sigma} \operatorname{tr}\left(\gamma^{\sigma}\left[T \otimes I_{E}\right](A)\right)\right\|_{L_{E}^{2}(\Omega)} \\
& =d_{\sigma}^{-1 / 2} \mathcal{K}_{22}(E, \mathcal{G})\left\|d_{\sigma} \operatorname{tr}\left(T^{\star}\left(\gamma^{\sigma}\right) A\right)\right\|_{L_{E}^{2}(\Omega)} \\
& =d_{\sigma}^{-1 / 2} \mathcal{K}_{22}(E, \mathcal{G})\left\|d_{\sigma} \operatorname{tr}\left(\gamma^{\sigma} A\right)\right\|_{L_{E}^{2}(\Omega)} \\
& \leq \mathcal{K}_{22}(E, \mathcal{G}) \mathcal{K}_{12}(E, \mathcal{G})\|A\|_{S_{d_{\sigma}}^{2}(E)}
\end{aligned}
$$

since, by the unitarity of $T$, the distribution of $T\left(\gamma^{\sigma}\right)$ is the same as that of $\gamma^{\sigma}$ (see Theorem 6.8 in Chapter 3 of [1]). Therefore $E$ satisfies (5).

Let $\Phi$ be a quantized orthonormal system and let $E$ be an operator space. Let $1 \leq p \leq 2$. We shall say that $E$ has Banach $\Phi$-type $p$ if

$$
\widetilde{\mathcal{K}}_{1 p}(E, \Phi)=\sup \left\|\mathcal{F}_{\Phi_{0}}^{-1} \otimes I_{E}\right\|_{\mathcal{B}\left(\mathcal{L}_{E}^{p}\left(\Sigma_{0}\right), \Phi_{E}^{p^{\prime}}\left(\Sigma_{0}\right)\right)}<\infty
$$

where the supremum is taken over the family of finite subsets $\Sigma_{0}$ of $\Sigma$. That is, we do not require the complete boundedness of $\mathcal{F}_{\Phi_{0}}^{-1} \otimes I_{E}$ as we did in Definition 2.4; we just require its boundedness. In the same fashion one can define the Banach $\Phi$-cotype $p^{\prime}$ of an operator space and the corresponding constant $\widetilde{\mathcal{K}}_{2 p^{\prime}}(E, \Phi)$. The following result, which is a consequence of the probabilistic argument employed in the proof of Theorem 5.6, shows that the notions of Banach $\Phi$-type and Banach $\Phi$-cotype 2 are the right ones in the operator space version of Kwapien's theorem whenever the quantized system $\Phi$ takes values in arbitrarily large matrices.

Corollary 5.7. Let $\mathbf{d}_{\Sigma}$ be an unbounded family of positive integers indexed by $\Sigma$. Let $\Phi$ be any u.b.q.o.s. with parameters $\left(\Sigma, \mathbf{d}_{\Sigma}\right)$. Let $E$ be an operator space. Then the following are equivalent:

(i) E is completely isomorphic to some $\mathrm{OH}$ Hilbertian operator space.

(ii) E has Banach $\Phi$-type and Banach $\Phi$-cotype 2.

(iii) E has Banach $\mathcal{G}$-type and Banach $\mathcal{G}$-cotype 2.

Proof. The implication (i) $\Rightarrow$ (ii) is obvious, and (ii) $\Rightarrow$ (iii) follows from Proposition 3.5 and the probabilistic proof of Theorem 5.6. Recall that the proofs of both results are still valid when complete boundedness is replaced by boundedness. Finally, (iii) $\Rightarrow$ (i) since the proof of Theorem 5.6 only uses the fact that $E$ has Banach $\mathcal{G}$-type and Banach $\mathcal{G}$-cotype 2 .

REMARK 5.8. Obviously this result fails for $\mathbf{d}_{\Sigma}$ bounded. For instance, take $\Phi$ to be the classical Rademacher system on $L^{2}[0,1]$ or the dual group of the torus $\mathbb{T}$. In these cases we go back to Kwapień's classical characterization of Hilbert spaces.

We now extend Corollary 5.7 to the case of complete quantized orthonormal systems with $\mathbf{d}_{\Sigma}$ unbounded. The proof of this result was kindly communicated to us by Gilles Pisier. 
THeOREM 5.9. Let $\mathbf{d}_{\Sigma}$ be an unbounded family of positive integers indexed by $\Sigma$. Let $\Psi$ be any complete quantized orthonormal system with parameters $\left(\Sigma, \mathbf{d}_{\Sigma}\right)$. Let $E$ be an operator space. Then the following are equivalent:

(i) E is completely isomorphic to some $\mathrm{OH}$ Hilbertian operator space.

(ii) E has Banach $\Psi$-type and Banach $\Psi$-cotype 2.

Proof. The implication (i) $\Rightarrow($ ii) is again obvious. To see that (ii) $\Rightarrow(\mathrm{i})$, we begin by recalling that if $E$ has Banach $\Psi$-type and Banach $\Psi$-cotype 2 , then (the underlying Banach space of) $E$ is isomorphic to a Hilbert space. That is, the proof of Theorem 4.2 can easily be adapted to this setting. Moreover, by Kwapień's another well known characterization given in [7], there exists a positive constant $c$ such that for any linear mapping $L: L^{2}(\Omega) \rightarrow L^{2}(\Omega)$, we have $\left\|L \otimes I_{E}\right\|_{\mathcal{B}\left(L_{E}^{2}(\Omega), L_{E}^{2}(\Omega)\right)} \leq c\|L\|_{\mathcal{B}\left(L^{2}(\Omega), L^{2}(\Omega)\right)}$. In particular, if $\Lambda^{2}$ is any closed subspace of $L^{2}(\Omega)$ and $\Lambda^{2}(E)=\Lambda^{2} \otimes E$, we get

$$
\left\|L \otimes I_{E}\right\|_{\mathcal{B}\left(\Lambda^{2}(E), \Lambda^{2}(E)\right)} \leq c\|L\|_{\mathcal{B}\left(\Lambda^{2}, \Lambda^{2}\right)}
$$

for any linear mapping $L: \Lambda^{2} \rightarrow \Lambda^{2}$. Now, for any $\sigma \in \Sigma$, we consider the space $\Lambda_{\sigma}^{2}=\operatorname{span}\left\{\psi_{i j}^{\sigma}: 1 \leq i, j \leq d_{\sigma}\right\}$ regarded as a subspace of $L^{2}(\Omega)$, and the space $\Lambda_{\sigma}^{2}(E)=\Lambda_{\sigma}^{2} \otimes E$. We also need to consider the linear isomorphism

$$
T_{2}(\sigma): S_{d_{\sigma}}^{2} \rightarrow \Lambda_{\sigma}^{2}, \quad A \mapsto d_{\sigma} \operatorname{tr}\left(A \psi^{\sigma}\right) .
$$

The following estimates are clear:

$$
\begin{aligned}
\left\|T_{2}(\sigma) \otimes I_{E}\right\|_{\mathcal{B}\left(S_{d_{\sigma}}^{2}(E), \Lambda_{\sigma}^{2}(E)\right)} & \leq d_{\sigma}^{1 / 2} \widetilde{\mathcal{K}}_{12}(E, \Psi), \\
\left\|T_{2}(\sigma)^{-1} \otimes I_{E}\right\|_{\mathcal{B}\left(\Lambda_{\sigma}^{2}(E), S_{d_{\sigma}}^{2}(E)\right)} & \leq d_{\sigma}^{-1 / 2} \widetilde{\mathcal{K}}_{22}(E, \Psi) .
\end{aligned}
$$

Finally, for any linear mapping $T: S_{d_{\sigma}}^{2} \rightarrow S_{d_{\sigma}}^{2}$, we have $T=T_{2}(\sigma)^{-1} \circ$ $L_{2}(\sigma) \circ T_{2}(\sigma)$ where $L_{2}(\sigma)=T_{2}(\sigma) \circ T \circ T_{2}(\sigma)^{-1}$ satisfies inequality (6). Therefore

$$
\begin{aligned}
\left\|T \otimes I_{E}\right\|_{\mathcal{B}\left(S_{d_{\sigma}}^{2}(E), S_{d_{\sigma}}^{2}(E)\right)} & \leq\left\|T_{2}(\sigma)^{-1} \otimes I_{E}\right\|\left\|L_{2}(\sigma) \otimes I_{E}\right\|\left\|T_{2}(\sigma) \otimes I_{E}\right\| \\
& \leq c \widetilde{\mathcal{K}}_{12}(E, \Psi) \widetilde{\mathcal{K}}_{22}(E, \Psi)\left\|L_{2}(\sigma)\right\|_{\mathcal{B}\left(\Lambda_{\sigma}^{2}, \Lambda_{\sigma}^{2}\right)} \\
& \leq c \widetilde{\mathcal{K}}_{12}(E, \Psi)^{2} \widetilde{\mathcal{K}}_{22}(E, \Psi)^{2}\|T\|_{\mathcal{B}\left(S_{d_{\sigma}}^{2}, S_{d_{\sigma}}^{2}\right)} .
\end{aligned}
$$

But then the hypothesis of Lemma 5.5 is satisfied since $\mathbf{d}_{\Sigma}$ is unbounded.

REMARK 5.10. In fact, it can be checked that the ideas behind the proof of Theorem 5.9 also yield Corollary 5.7. In particular, the probabilistic approach given at the beginning of this section is not necessary to get Corollary 5.7. However, we have included it since we consider it as a natural source of ideas for these results. 
Let $R$ and $C$ denote the row and column operator spaces respectively. In [12] Pisier defined natural operator space structures on $R \cap C$ and $R+C$ in such a way that the pair $(R \cap C, R+C)$ becomes compatible for complex interpolation. Moreover, Pisier proved in [13] the following surprising complete isomorphism:

$$
(R \cap C, R+C)_{\theta} \simeq \mathrm{R}_{p},
$$

where $\theta=1 / p$ and $\mathrm{R}_{p}$ is (as in Remark 3.3) the closure in $L^{p}[0,1]$ of the subspace spanned by the classical Rademacher functions $r_{1}, r_{2}, \ldots$ endowed with its natural operator space structure. Pisier [13] analyzed this operator space structure by means of the non-commutative Khinchin inequalities previously developed by him and Lust-Piquard (see [8] and [9]). Now we use the family $\left\{\mathrm{R}_{p}: 1 \leq p \leq \infty\right\}$ of operator spaces to illustrate some situations:

$\left(\mathrm{a}_{1}\right)$ Let $\Phi$ be any u.b.q.o.s. associated to the parameters $\left(\Sigma, \mathbf{d}_{\Sigma}\right)$ with $\mathbf{d}_{\Sigma}$ unbounded. Then $\mathrm{R}_{p}$ has Banach $\Phi$-type 2 for any $2 \leq p<\infty$. Namely, by the classical Khinchin inequalities the underlying Banach space of $\mathrm{R}_{p}$ is isomorphic to that of $\mathrm{R}_{2}$ for $1 \leq p<\infty$. Moreover, the identity mapping $I: \mathrm{R}_{p} \rightarrow \mathrm{R}_{2}$ is a complete contraction whenever $p \geq 2$. Therefore, there exists some constant $c$ such that

$$
\begin{aligned}
\left\|\sum_{\sigma \in \Sigma_{0}} d_{\sigma} \operatorname{tr}\left(A^{\sigma} \varphi^{\sigma}\right)\right\|_{L_{\mathrm{R}_{p}}^{2}(\Omega)} & \leq c\left\|\sum_{\sigma \in \Sigma_{0}} d_{\sigma} \operatorname{tr}\left(A^{\sigma} \varphi^{\sigma}\right)\right\|_{L_{\mathrm{R}_{2}}^{2}(\Omega)} \\
& \leq c \widetilde{\mathcal{K}}_{12}\left(\mathrm{R}_{2}, \Phi\right)\left(\sum_{\sigma \in \Sigma_{0}} d_{\sigma}\left\|A^{\sigma}\right\|_{S_{d_{\sigma}}^{2}\left(\mathrm{R}_{2}\right)}^{2}\right)^{1 / 2} \\
& \leq c \widetilde{\mathcal{K}}_{12}\left(\mathrm{R}_{2}, \Phi\right)\left(\sum_{\sigma \in \Sigma_{0}} d_{\sigma}\left\|A^{\sigma}\right\|_{S_{d_{\sigma}}^{2}\left(\mathrm{R}_{p}\right)}^{2}\right)^{1 / 2} .
\end{aligned}
$$

Now Corollary 5.7 shows that $\mathrm{R}_{p}$, although isomorphic to a Hilbert space, cannot have Banach $\Phi$-cotype 2 for $2<p<\infty$ since in that case $\mathrm{R}_{p}$ is not completely isomorphic to any $\mathrm{OH}$ operator space. By Theorem 5.9, the same holds when we work with any complete quantized orthonormal system $\Psi$ with $\mathbf{d}_{\Sigma}$ unbounded.

$\left(\mathrm{a}_{2}\right)$ Similarly $\mathrm{R}_{p}$ has Banach $\Phi$-cotype 2 for any $1 \leq p \leq 2$ but it does not have Banach $\Phi$-type 2 unless $p=2$. By Theorem 5.9, the same holds for any complete quantized orthonormal system $\Psi$ with $\mathbf{d}_{\Sigma}$ unbounded.

(b) In the commutative theory there exist some systems for which Kwapień's theorem holds if we require only one of the type 2 or cotype 2 conditions. Kwapień showed in [6] that the system of characters of the torus $\mathbb{T}$ presents this kind of autoduality. Another example is given by the system of characters of the Cantor group $\mathbb{D}$ (see [3] or [11] for a proof of this fact). It is easy to see that this autoduality remains valid in our set- 
ting. For instance, if $E$ has $\mathbb{Z}$-type 2, then so does $S^{2}(E)$ and hence it is isomorphic to some Hilbert space $H$. But this implies that $E$ is completely isomorphic to some $\mathrm{OH}$ (see the proof of Theorem 3.10). In particular $\mathrm{R}_{p}$ cannot have Fourier type 2 or Fourier cotype 2 with respect to $\mathbb{T}$ or $\mathbb{D}$ unless $p=2$. On the other hand, we know that $\mathrm{R}_{p}$ has BanachFourier type and Banach-Fourier cotype 2 with respect to $\mathbb{T}$ and $\mathbb{D}$ for any $1 \leq p<\infty$.

Now, it is natural to ask if there exists a non-commutative compact group $G$ with dual object $\Gamma$ satisfying this autoduality, that is, such that any operator space $E$ having $\Gamma$-type 2 or $\Gamma$-cotype 2 is completely isomorphic to some $\mathrm{OH}$ operator space. At least we know that when $\mathbf{d}_{\Gamma}$ is unbounded, by points $\left(\mathrm{a}_{1}\right)$ and $\left(\mathrm{a}_{2}\right)$, an operator space having Banach $\Gamma$-type 2 or Banach $\Gamma$-cotype 2 does not have to be completely isomorphic to any $\mathrm{OH}$ operator space.

At this point it also becomes natural to ask if Banach $\Gamma$-type 2 and $\Gamma$-type 2 (resp. Banach $\Gamma$-cotype 2 and $\Gamma$-cotype 2 ) are equivalent notions as a consequence of the unboundedness of $\mathbf{d}_{\Gamma}$. At the time of this writing, we are not able to answer these questions.

\section{References}

[1] A. Araujo and E. Giné, The Central Limit Theorem for Real and Banach Valued Random Variables, Wiley Ser. Probab. Statist., Wiley, 1980.

[2] E. G. Effros and Z. J. Ruan, Operator Spaces, London Math. Soc. Monogr. 23, Oxford Univ. Press, 2000.

[3] J. García-Cuerva, K. Kazarian, V. Kolyada and J. L. Torrea, Vector-valued Hausdorff-Young inequality and applications, Russian Math. Surveys 53 (1998), 435-513.

[4] J. García-Cuerva, J. M. Marco and J. Parcet, Sharp Fourier type and cotype with respect to compact semisimple Lie groups, Trans. Amer. Math. Soc. (2003), to appear.

[5] J. García-Cuerva and J. Parcet, Fourier analysis and operator spaces: Vector-valued Hausdorff-Young inequality on compact groups, submitted for publication (2003).

[6] S. Kwapień, Isomorphic characterizations of inner product spaces by orthogonal series with vector valued coefficients, Studia Math. 44 (1972), 583-595.

[7] -, On operators factorizable through $L_{p}$-spaces, Bull. Soc. Math. France Mém. 31-32 (1972), 215-225.

[8] F. Lust-Piquard, Inégalités de Khintchine dans $C_{p}(1<p<\infty)$, C. R. Acad. Sci. Paris 303 (1986), 289-292.

[9] F. Lust-Piquard and G. Pisier, Non-commutative Khintchine and Paley inequalities, Ark. Mat. 29 (1991), 241-260.

[10] M. B. Marcus and G. Pisier, Random Fourier Series with Applications to Harmonic Analysis, Ann. of Math. Stud. 101, Princeton Univ. Press, 1981.

[11] A. Pietsch and J. Wenzel, Orthonormal Systems and Banach Space Geometry, Encyclopedia Math. Appl. 70, 1998. 
[12] G. Pisier, The operator Hilbert space OH, complex interpolation and tensor norms, Mem. Amer. Math. Soc. 122 (1996).

[13] - Non-commutative vector valued $L_{p}$-spaces and completely $p$-summing maps, Astérisque 247 (1998).

Departamento de Matemáticas, C-XV

Universidad Autónoma de Madrid

28049 Madrid, Spain

E-mail: jose.garcia-cuerva@uam.es javier.parcet@uam.es 